\title{
Congruence of decay chains of elements 113, 115 , and 117
}

\author{
Ulrika Forsberg ${ }^{\text {, }}$ Claes Fahlander, and Dirk Rudolph \\ Department of Physics, Lund University, 22100 Lund, Sweden
}

\begin{abstract}
Superheavy element research relies heavily on the detection of correlated decay chains. When several chains have been detected, it is natural to ask whether they form one congruent set, or whether they should be further divided into subsets. To statistically assess the congruence between the chains in a data set, we developed a procedure to calculate a Figureof-Merit for low-number statistics. In this work, the method is applied to the world data set of odd- $Z$ superheavy nuclei with $Z \geq 113$. Some of the postulated sets of chains are found to be congruent, while others are not. The findings are of relevance for the IUPAC and IUPAP approvals of the new elements $Z=113,115$, and 117 .
\end{abstract}

\section{Introduction}

Superheavy nuclei assumed to have atomic numbers $Z=113,115$, and 117 , have been produced in heavy-ion fusion-evaporation reactions, and implanted into charged-particle detectors [1-10]. Superheavy elements often decay by subsequent $\alpha$ emission and thus form decay chains. These chains are often terminated by spontaneous fission, and they are found by searching for correlations in the data sets. A chain is characterized by its length and the types of decays that are observed, together with the decay energy and correlation time measured in each of the steps. Commonly, several different isotopes are expected to be produced in the same experiment. The chains can sometimes be divided into groups according to their characteristics, and then these groups can eventually be assigned to different isotopes. It is difficult to assess whether groups of chains are congruent, i.e., whether the chains are correctly grouped, or not. The advantages and disadvantages of various characteristics of decay chains led us to develop a low-statistics method, in which a Figure-of-Merit based on experimentally measured lifetimes is compared with the Figure-of-Merit of Monte-Carlo generated chains [11]. In this manuscript, the method is further motivated and applied to groups of the heaviest odd- $Z$ superheavy nuclei. The results are of relevance to the IUPAC and IUPAP decisions to accept the new elements with $Z=113,115$, and 117 .

\section{Decay chain characteristics}

Each of the characteristics of chains (length, decay modes, energies, and times) can be used to classify and divide them into different groups. However, any of these characteristics poses its own difficulty. The chain length and the decay modes are not necessarily good descriptors, since various decay modes can be present in the same nucleus. Spontaneous fission, $\alpha$ decay,

\footnotetext{
${ }^{a}$ Corresponding author: ulrika.forsberg@nuclear.lu.se
}

(C) The Authors, published by EDP Sciences. This is an Open Access article distributed under the terms of the Creative Commons Attribution License 4.0 (http://creativecommons.org/licenses/by/4.0/). 
and electron capture (EC) decay branches are predicted to co-exist in many superheavy nuclei (see, for example, Ref. [12]). Here, it is also of interest to note that none of the detector setups employed in experiments on elements $Z=113,115$, and 117 have been sensitive to EC.

The $\alpha$-decay energies can provide good characterisation of a chain if the decays always proceed to the same state in the daughter nucleus. This is usually the case for even-even nuclei. For odd-even, even-odd, or odd-odd nuclei, decays from one nucleus often proceed to several different states in the daughter. Such $\alpha$ decays give rise to a variety of different measured $\alpha$ energies. Hence, differing energies do not necessarily imply that the chains should be assigned to different isotopes.

In the cases where the $\alpha$ decays proceed to excited states in the daughter nucleus, there are additional aspects to keep in mind. If the excited state de-excites to a lower state by emission of electrons from internal conversion (IC), a partial (or full) summing of the electron and $\alpha$ energies may occur. This leads to a smearing of the otherwise discrete $\alpha$ peaks [13]. If, on the other hand, the excited state does not de-excite but rather undergoes $\alpha$ decay directly, other difficulties arise. The average lifetime for $\alpha$ decays starting from an excited state is not the same as the average lifetime for decays starting from the ground state. Therefore, a particular decay step associated with one isotope can decay with several different lifetimes. Furthermore, nuclear structure calculations (see, e.g., Ref. [11]) suggest that in some isotopes, $\alpha$ decays from different states in one nucleus lead to the population of different states also in the daughter nucleus. Therefore, decays starting from the same isotope can produce differentlooking decay sequences.

Although there is no certain correspondence between different-looking decay sequences and different isotopes, the basic question of how we can test the congruence within a group remains. The measured times for decays proceeding from a particular state in a chain are observations of a random variable described by an exponential distribution. Thus, the decay times are not necessarily similar to each other. If the spread of the times in a data set is too small, the data has probably been divided into too many subsets. If, on the other hand, the spread is too large, the data probably originates from more than one exponential decay, and the data set must be further divided into subsets. In Ref. [14], K.-H. Schmidt characterized the spread as the standard deviation of the times, and used sets of Monte-Carlo generated exponentially distributed times to calculate 1- $\sigma$ confidence limits for the standard deviation. This test applies to one decay step at the time. In Ref. [15], we proposed a generalisation of this method, where the entire chains can be taken into account. A similar approach was presented in Ref. [11], and it is this version of the method that we will use in this work. In short, a probability density function, based on the average lifetime and its uncertainty, is constructed for each decay step. The function is evaluated for each measured lifetime, and the result is taken as a Figure-of-Merit (FoM). The FoM for a chain is defined as the geometrical average over the individual FoMs in this chain. The FoM for a group of chains is defined as the arithmetic average of the FoMs of all the chains. The FoM for the data set is compared with the expected distribution for the FoM. The expected distribution is found by calculating the FoM for sets of Monte-Carlo generated chains that do fulfill the hypothesis of a common origin.

\section{Available data sets for superheavy nuclei with $Z=113,115$ and 117}

To date, odd- $Z$ superheavy elements with $Z \geq 113$ have been directly produced in the reaction ${ }_{30} \mathrm{Zn}+{ }_{83} \mathrm{Bi}$ and in ${ }_{20}^{48} \mathrm{Ca}$-induced reactions on ${ }_{93} \mathrm{~Np}$, ${ }_{95} \mathrm{Am}$, and ${ }_{97} \mathrm{Bk}$. The number 
Table 1. Chains of superheavy nuclei with $Z=113,115$, and 117 . The chains from each element have been divided into subsets according primarily to their lengths. In some cases, an isotope assignment is given. The chains are also sorted according to the laboratory where they were observed: JINR (Russia), GSI (Germany), LBNL (USA), and RIKEN (Japan).

JINR GSI LBNL RIKEN total

\begin{tabular}{lccccc}
\hline "long" E117 $\left({ }^{294} 117\right)$ & 4 & 2 & - & - & 6 \\
"short" E117 $\left({ }^{293} 117\right)$ & 16 & - & - & - & 16 \\
${ }^{287} 115$ & 2 & 1 & - & - & 3 \\
${ }^{288} 115$ & 31 & 22 & 43 & - & 96 \\
"short" 115 & 4 & 7 & 3 & - & 14 \\
278113 & - & - & - & 3 & 3 \\
282113 & 2 & - & - & - & 2 \\
\hline
\end{tabular}

of chains are summarized in Table 1. Of element 113, three chains have been produced at RIKEN [2-4] and two chains have been produced at JINR [5]. The RIKEN chains have been assigned to the isotope ${ }^{278} 113$ and the JINR chains to ${ }^{282} 113$. Of element 115 , a total of 113 decay chains has been observed. The chains have coarsely been divided into three different groups - three long chains (five- $\alpha$ ) with rather short lifetimes in all steps, 96 long chains (five$\alpha$ ) with relatively longer lifetimes, and fourteen "short" one- $\alpha$ and two- $\alpha$ decay chains. These three groups of chains have been assigned to the isotopes ${ }^{287} 115,{ }^{288} 115$, and ${ }^{289} 115$ in, e.g., Ref. [6]. Of element 117, twenty-two chains have been observed. Six of them consisted of six $\alpha$ decays and will be referred to as "long". Sixteen of the chains consisted of three or four $\alpha$ decays and will be referred to as "short". In, e.g., Ref. [7], the long chains have been assigned to the isotope ${ }^{294} 117$ and the short chains to ${ }^{293} 117$. Hence, the short element 117 chains are supposed to feed the isotope ${ }^{289} 115$, and it is of interest to also evaluate the congruence in the joint set of these chains.

\section{Statistical assessments of element $\mathbf{1 1 3}$ decay chains}

The proposed statistical test has been applied to the three $\alpha$-decay chains assigned to start from ${ }^{278} 113$ [2-4]. Among these chains, two consist of five decay steps, and one chain consists of six decay steps. There are no missing members in the chains, i.e., all the lifetimes have been measured. The test is performed on the first five decay steps only, as there is only one chain which has more members. The FoM value for the experimental data set was 0.155 . To obtain a reference distribution for the FoM, $10^{5}$ sets - each consisting of three chains with five decay steps - were Monte-Carlo generated. The uncertainty in the lifetime in each step was taken into account by generating the lifetimes from different exponential distributions, where the inverse decay constant $\tau$ was selected from the appropriate likelihood function (given the average lifetime and the number of available lifetimes). The FoM was calculated for each of the $10^{5}$ generated data sets, and the distribution was characterized by the $90 \%$ and 98\% double-sided confidence intervals. These intervals are shown in Table 2. The FoM for the experimental data set $(0.155)$ fits within the $90 \%$ confidence interval. Hence, from this perspective, there is no reason to question the common origin of these three chains.

It is interesting to note that the original Schmidt test [14] would yield the result that decay steps one, two, and four have standard deviations $(1.13,0.601$, and 0.57 , respectively) that are all within the respective 1- $\sigma$ interval $[0.19,1.91]$, while decay steps three and five have standard deviations $(2.31,2.18$, respectively) that are outside this interval on the upper side. However, when all steps are considered in the new test, the conclusion is that the data set is congruent. These two views can still be in agreement: The low level of confidence associated with one $\sigma$ means that the standard deviations should be outside the confidence intervals 
Table 2. Experimental and reference values for the FoM. The experimental data set which is modeled by the Monte-Carlo generated chains, the FoM for this data set, the number of chains, the number of decay steps that are considered, and the double-sided $90 \%$ and $98 \%$ confidence intervals, are presented. The limits have been obtained from FoM distributions from $10^{5}$ Monte-Carlo generated chains. See text and references $[11,16,17]$ for details.

\begin{tabular}{lccccc} 
& Exp. FoM & No. chains & No. steps & $90 \%$ & $98 \%$ \\
\hline${ }^{278} 113$ & 0.155 & 3 & 5 & {$[0.115,0.226]$} & {$[0.094,0.244]$} \\
${ }^{288} 115$ & 0.224 & 96 & 6 & {$[0.195,0.221]$} & {$[0.189,0.226]$} \\
${ }^{287} 115$ & 0.225 & 3 & 6 & {$[0.111,0.214]$} & {$[0.090,0.231]$} \\
"long" E117 $\left({ }^{294} 117\right)$ & 0.226 & 6 & 6 & {$[0.144,0.234]$} & {$[0.126,0.250]$} \\
"short" E117 $\left({ }^{293} 117\right)$ & 0.173 & 16 & 4 & {$[0.173,0.244]$} & {$[0.158,0.257]$} \\
all "short" E117 and E115 & 0.153 & 30 & 3 & {$[0.187,0.244]$} & {$[0.175,0.254]$} \\
\hline
\end{tabular}

in some of the decay steps even in a congruent data set. Moreover, the new test is slightly more "generous" due to the inclusion of lifetime uncertainties when the reference chains are generated, and the use of an average lifetime that is not tailored to each of the generated data sets (see Ref. [16]). This translates to a situation where experimental sets of decay chains are rather accepted as congruent than discarded as consisting of more than one radioactive source.

\section{Statistical assessments of element 115 decay chains}

The statistical method has been applied to the data set containing 96 five- $\alpha$ long decay chains assigned to the isotope ${ }^{288} 115$ in Ref. $[6,8,9] .10^{5}$ sets of 96 chains were Monte-Carlo generated. To mimic the detailed structure of the chains, i.e. to take into account that some members in the chains were missing, the Monte-Carlo generated sets of numbers where pruned accordingly. The FoM for the data set is 0.224 . The different confidence intervals are presented in Table 2. The experimental FoM falls within the $98 \%$ confidence interval, but outside the $90 \%$ interval, on the upper side. This suggests that the measured lifetimes fit almost too well. However, as pointed out in the previous section, the test has been constructed such that congruence is rather exaggerated than understated. Therefore, the high FoM does not mean that the lifetimes are necessarily too similar. Although the test is inconclusive in this case, the reasons for a high FoM could be that the measurements have been insensitive to either (or both) very short or very long lifetimes, that some of the remaining chains observed in the ${ }^{48} \mathrm{Ca}$ on ${ }^{243} \mathrm{Am}$ reaction should be included in this group rather than in the other two groups associated with element 115 , and/or that uncertain identification of chain members have led to the exclusion of the member rather than tentative inclusion (see, e.g., Table 1 in the Supplemental Material of Ref. [8], where it is indicated that there are "several options").

When the test is applied to the three five- $\alpha$ long chains assigned to start from the isotope ${ }^{287} 115$, the resulting FoM is 0.225 . This value falls within the $98 \%$ confidence interval, but outside the $90 \%$ interval, on the upper side (see Table 2), which indicates that the similarities between the times are almost too good also in this case.

In Ref. [11], the test was applied to the fourteen "short" (one- $\alpha$ and two- $\alpha$ ) element 115 chains. In brief, the set of all fourteen chains is not congruent. The FoM is well below the $98 \%$ double-sided confidence interval. Hence, the conclusion that the data set is not congruent has an error probability of less than $1 \%$. However, several different subsets of the chains are internally congruent. It is suggested that some of the short chains should rather be assigned to the isotope ${ }^{288} 115$, which would lead to spontaneous fission branches in ${ }^{284} 113$ and ${ }_{111}^{280} \mathrm{Rg}-$ or, as discussed in detail in Ref. [11], to undetected electron capture branches with subsequent spontaneous fission from the even-even daughters ${ }^{284} \mathrm{Cn}$ and ${ }^{280} \mathrm{Ds}$, respectively. 


\section{Statistical assessments of element 117 decay chains}

The test was applied to the six "long" element 117 chains. The experimental FoM of 0.226 is within the $90 \%$ confidence interval (see Table 2). Hence, this is a congruent data set. The test was also applied to the sixteen "short" element 117 chains. Although two of the chains have one more member, only four decay steps were considered. The resulting FoM is 0.173 - just on the lower limit of the $90 \%$ confidence interval (see Table 2). In Ref. [17], the congruence of a subset of ten of the sixteen "short" (three- $\alpha$ and four- $\alpha$ ) element 117 chains was investigated. The subset was selected in accordance with Ref. [18], and contains those chains in which there are no missing members. The resulting FoM of 0.165 was between the $1 \%$ and $5 \%$ lower confidence limits. This indicates that the measured lifetimes, in both the full and the restricted data set, are too different from each other and that the data sets most likely should be divided into subsets.

\section{Statistical assessments of the alleged link between element 115 and element 117}

Assuming that the short element 117 chains originate from the isotope ${ }^{293} 117$ and that the short element 115 chains originate from the isotope ${ }^{289} 115$, the $\alpha$ decay of the former should feed the latter. In such case, these data sets could be combined into one. The statistical test was applied in Ref. [17] to the three decay steps E115 $\rightarrow$ E113 $\rightarrow$ E111 in such a combined data set. From element 117, the ten element 117 chains which had no missing members were used, and from element 115, the four chains observed at JINR. This set had a FoM which was below both the $90 \%$ and the $98 \%$ confidence interval, i.e., the error probability is less than $1 \%$ for the conclusion that this is not a congruent data set. This lack of congruence is important because the IUPAC and IUPAP approvals of the new elements 115 and 117 are based on Ref. [18], in which the postulated congruence of this data set poses the only argument for the discovery of these elements.

Further, the full data set on short element 115 and 117 chains - all fourteen short element 115 decay chains and all sixteen short element 117 decay chains - was investigated. The resulting FoM is 0.153 , which is well below the $98 \%$ confidence interval (see Table 2). This means that inclusion of the subsequently acquired data from GSI and LBNL, and of element 117 chains which had missing members, does not improve the likeliness of this link.

\section{Further comments on Ref. [18]}

The approval of elements 115 and element 117 rely on a link between these elements that is, as shown by our statistical test, with a very high probability non-existent. This is problematic. Furthermore, there are other scientific inaccuracies in Ref. [18]. Some of these have already been discussed in Ref. [17]. Here, a selection of further aspects of the report are discussed in some detail.

As pointed out correctly in Ref. [18], $\alpha$ decays in odd-odd, odd-even, and even-odd nuclei tend to proceed to various states in the daughter nucleus (see Fig. 1). Hence, extreme care should be taken before attempting to calculate average $\alpha$ energies. If the measured energies correspond to different transitions, the average of these energies is a meaningless quantity. Possibly, it could be used to check consistency between subsequent measurements, in cases where the number of events is large enough that the branching ratios to different excited states are accurately reproduced. This is hardly the case for the reported measurements on elements 113,115 , and 117 . Nonetheless - although data for single chains are readily available, and 


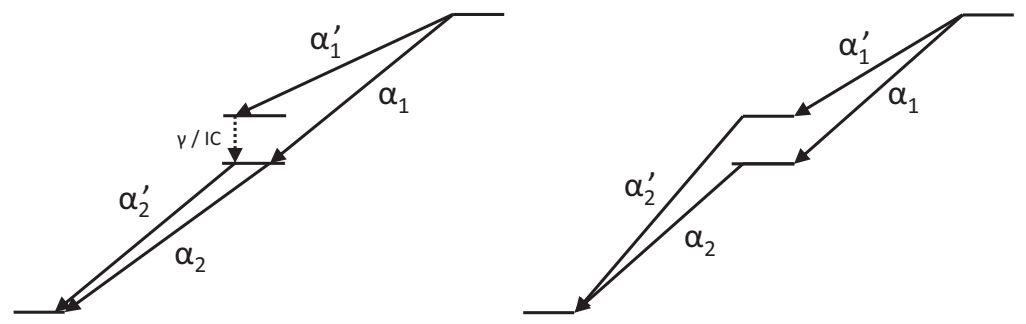

Figure 1. Schematic picture over two possible decay scenarios of a nuclide. The $\alpha$ decays proceed to different states in the intermediate nucleus. The populated excited states de-excites via emission of $\gamma$ rays or internal conversion, and the subsequent $\alpha$ decays proceed from ground state to ground state (left). The populated excited state undergoes $\alpha$ decay to the ground state in the grand daughter (right).

although the authors of Ref. [18] acknowledge that the presence of transitions to different states has been demonstrated - such averages are used throughout the article.

Furthermore, the sum of $\alpha$ energies along decay chains is presented as a quantity that can shed light on the congruence between different chains. The anticipated physical background is shown in Fig. 1 (right) for chains consisting of two steps. The $\alpha$ decays from the mother isotope proceed to either the ground state or an excited state in the daughter nucleus. Then, the next $\alpha$ decays occur from the respective state to the ground state in the grand-daughter nucleus. Although the decay energies $\alpha_{1}$ and $\alpha_{1}^{\prime}$ are not the same, and also $\alpha_{2}$ and $\alpha_{2}^{\prime}$ are not the same, the summed energies $\alpha_{1}+\alpha_{2}$ and $\alpha_{1}^{\prime}+\alpha_{2}^{\prime}$ are equal. It is argued that if the sums are similar, it indicates that the chains start and end in the same nuclear states and, thus, that the chains do share a common origin.

This argumentation is valid as long as no $\gamma$ rays or internal conversion electrons are emitted. Such de-excitations are most likely present in most odd-nuclei decays, and their presence has been firmly established in chains assigned to start from the isotope ${ }^{288} 115[8,9]$. If the sum- $\alpha$ energies agree for different chains although $\gamma$ rays and internal conversions have not been excluded, this might be due to pure chance. Several hundreds of $\mathrm{keV}$ could be missed in each step (see, e.g., the decay step ${ }^{276} \mathrm{Mt} \rightarrow{ }^{272} \mathrm{Bh}$ ) in one of the chains, while the decays in another (different-origin) chain might proceed from ground state to ground state. In this way, the sums can agree although this does not say anything about the origin of the chains. Naturally, the chances for such unfortunate coincidences between differentorigin chains decrease if the energies are very precisely measured: The chances that two decay sequences should have identical sums is small. However, the individual $\alpha$ energies in the decay chains assigned to the nuclide ${ }^{278} 113$ (where the sum-energy argument is proposed) are measured with an uncertainty of $\sim 40 \mathrm{keV}$ at best, according to Ref. [18].

Another interesting observation is that if a decay proceeds as in Fig. 1 (right), the $\alpha$ decays from the intermediate nucleus start from different nuclear states. Decays from two different nuclear states are characterized by two different decay constants, i.e., they exhibit two different lifetimes. This means that for subsequent decay steps where such behaviour has been established, the similarities in lifetimes become meaningless quantities that must not be used to (artificially) reinforce an energy-summing argument.

Yet another aspect of identification of superheavy elements that becomes important especially for odd-nuclei, is that different states may be reached in a nucleus when it is created directly in a fusion-evaporation reaction and when it is created as a decay product. A prime example is ${ }^{257} \mathrm{Rf}$, where different decays sequences result when the isotope is directly produced and when it is created as the daughter of ${ }^{261} \mathrm{Sg}[19,20]$. Hence, the lack of an apparent link between the presumed isotopes ${ }^{289} 115$ and ${ }^{293} 117$ does not necessarily 
imply that the observed chains cannot originate from these isotopes. The lack of clear crossbombardment cases for odd nuclei is not unexpected.

\section{Summary}

In this manuscript, new applications of the statistical test introduced in Ref. [11] have been presented. Some data sets are found to be congruent in terms of decay times, while others have FoM values that indicate that a revision is needed. For some data sets the lifetimes fit "too well", which indicates that the selection of chains has maybe been too restrictive. In other cases the FoM value is low, which indicates that the chains do not share a common radioactive origin. In particular, the alleged cross-bombardment link between element 117 and element 115 is, on a more than $2-\sigma$ level, absent. It is further exemplified that methods and numerical operations in Ref. [18] are questionable.

This work is supported by the Swedish Research Council (VR 2013-4271).

\section{References}

[1] Yu. Oganessian et al., Phys. Rev. C 69, 021601(R) (2004).

[2] K. Morita et al., J. Phys. Soc. Jpn. 73, 2593 (2004).

[3] K. Morita et al., J. Phys. Soc. Jpn. 76, 045001 (2007).

[4] K. Morita et al., J. Phys. Soc. Jpn. 81, 103201 (2012).

[5] Yu.Ts. Oganessian et al., Phys. Rev. C 76, 011601(R) (2007).

[6] Yu.Ts. Oganessian et al., Phys. Rev. C 87, 014302 (2013).

[7] Yu.Ts. Oganessian et al., Phys. Rev. C 87, 054621(2013).

[8] D. Rudolph et al., Phys. Rev. Lett. 111, 112502 (2013).

[9] J.M. Gates et al., Phys. Rev. C 92, 021301(R) (2015).

[10] J. Khuyagbaatar et al., Phys. Rev. Lett. 112, 172501 (2014).

[11] U. Forsberg et al., Nucl. Phys. A953 117 (2016).

[12] A.V. Karpov et al., Int. J. Mod. Phys. E 21, 1250013 (2012).

[13] F.P. Heßberger et al., Nucl. Instr. Meth. A274, 522 (1989).

[14] K.-H. Schmidt, Eur. Phys. J. A8, 141 (2000).

[15] D. Rudolph et al., EPJ Web of Conferences 117, 010001 (2016).

[16] U. Forsberg, PhD thesis, Lund University, ISBN 978-91-7623-813-4 (2016).

[17] U. Forsberg et al., Phys. Lett. B760, 293 (2016).

[18] P.J. Karol et al., Pure Appl. Chem. 88, 139 (2016).

[19] B. Streicher et al., Eur. Phys. J. A45, 275 (2010).

[20] F.P. Heßberger, these proceedings. 